\title{
Keeping together
}

Collective cell movement - needed for normal development but also associated with the invasion of cancer cells - requires organization and coordination. An actomyosin force around the edge of a migrating cell cluster facilitates coordinated movement, but contractility at cell-cell contacts inside the cluster must be reduced to maintain group cohesion. Insight into how this is achieved is outlined by Hidalgo-Carcedo et al. in Nature Cell Biology.

Newly plated single cells showed high myosin light chain (MLC) activity around their cortex; upon aggregation, activity remained high around the edge of clusters but was downregulated at sites of cell contact. Similarly, myosin Ila localized preferentially around the edges of migrating A431 cell clusters. Artificially increasing actomyosin activity caused uncoordinated cell

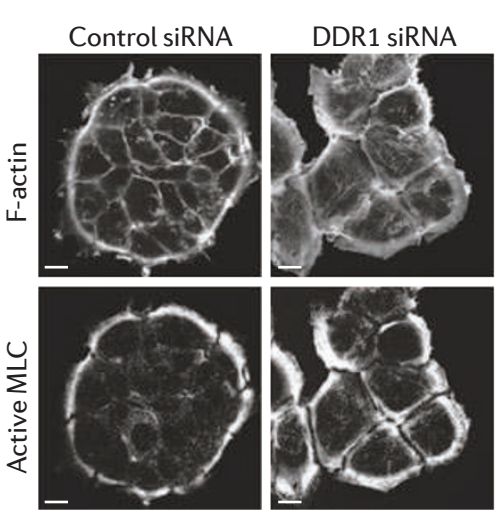

movement and breakaway clusters

of cells.

Next, Hidalgo-Carcedo et al. focused on discoidin domain receptor 1 (DDR1), as its depletion altered the organization of filamentous actin ( $\mathrm{F}$-actin) and caused myosin Ila and active MLC to localize to cell-cell contacts within clusters. The DDR1-depleted cells failed to move collectively in two-dimensional (2D), $3 \mathrm{D}$ and organotypic culture models.

Neither the collagen-binding activity nor the kinase domain of DDR1 was required to regulate $F$-actin organization at cell-cell contacts, where E-cadherin mediates DDR1 localization. Instead, DDR1 interacted, through its carboxyl terminus, with the PDZ domain of the cell polarity regulators PAR3 or PAR6. DDR1 and PAR3 colocalized at cell-cell contacts in invasive squamous cell and breast carcinomas and mutually regulated

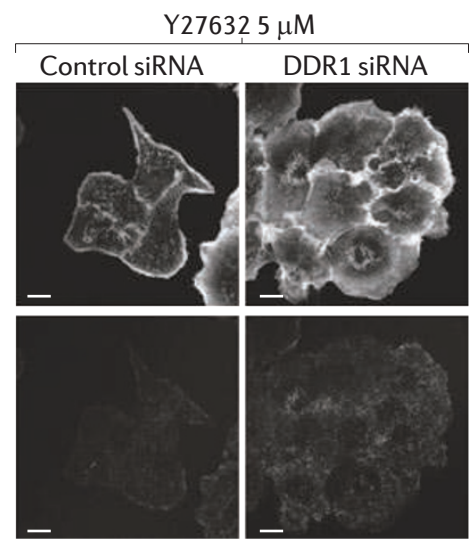

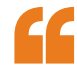

\section{DDR1 reduces actomyosin} activity at sites of cell-cell

\section{contact}

Depletion of discoidin domain receptor 1 (DDR1) leads to increased active myosin light chain (MLC) and altered filamentous actin (F-actin) at cell-cell contacts. Treatment with a RHO kinase inhibitor (Y27632) (right panels) in DDR1-depleted A431 squamous cell carcinoma cells restored F-actin organization and decreased MLC activity at cell-cell contacts (compared with untreated cells; left panels). siRNA, small interfering RNA. Image is reproduced, with permission, from Hidalgo-Carcedo, C. et al. (C) (2011) Macmillan Publishers Ltd. All rights reserved. each other's localization in A431 cells. Notably, the absence of either PAR3 or PAR6 resulted in the presence of active MLC at, and the loss of F-actin organization from, cell-cell contacts within clusters, preventing these cells from invading collectively.

Hidalgo-Carcedo et al. had, meanwhile, observed that depleting RHOE or p190ARHOGAP (also known as GRLF1) caused phenotypes similar to DDR1 depletion. RHOE and p190ARHOGAP can antagonize RHO kinase (ROCK)-mediated actomyosin contractility, and RHOE localizes to cell-cell contacts. The authors ascertained that DDR1 controls RHOE localization, although exactly how remains unclear. Subsequently, they showed that inhibiting ROCK in DDR1-depleted cells restored F-actin organization and decreased MLC activity at cell-cell contacts.

So, DDR1 reduces actomyosin activity at sites of cell-cell contact - thereby keeping migrating cell clusters together - by recruiting PAR3 (and/or PAR6, potentially) to these contacts; here, the DDR1-PAR3/PAR6 complex controls the localization of RHOE, which in turn antagonizes ROCK. As loss of cell polarity and reduced cell cohesion are two common features of cancer, it will be interesting to determine how the mechanisms described by Hidalgo-Carcedo et al. modulate tumour progression.

Katrin Legg, Gateway Assistant Editor Cell Migration Gateway

ORIGINAL RESEARCH PAPER HidalgoCarcedo, C. et al. Collective cell migration requires suppression of actomyosin at cell-cell contacts mediated by DDR1 and the cell polarity regulators Par3 and Par6. Nature Cell Biol. 13. 49-58 (2011) 